\title{
A Trajectory Estimation Method for Badminton Shuttlecock Utilizing Motion Blur
}

\author{
Hidehiko Shishido, Itaru Kitahara, Yoshinari Kameda, and Yuichi Ohta \\ University of Tsukuba \\ Tennoudai 1-1-1, Tsukuba, Ibaraki 305-8573, Japan \\ shishido@image.iit.tsukuba.ac.jp, \\ \{kitahara, kameda, ohta\}@iit.tsukuba.ac.jp
}

\begin{abstract}
To build a robust visual tracking method it is important to consider issues such as low observation resolution and variation in the target object's shape. When we capture an object moving fast in a video camera motion blur is observed. This paper introduces a visual trajectory estimation method using blur characteristics in the 3D space. We acquire a movement speed vector based on the shape of a motion blur region. This method can extract both the position and speed of the moving object from an image frame, and apply them to a visual tracking process using Kalman filter. We estimated the 3D position of the object based on the information obtained from two different viewpoints as shown in figure 1. We evaluated our proposed method by the trajectory estimation of a badminton shuttlecock from video sequences of a badminton game.
\end{abstract}

Keywords: Visual Object Tracking, Motion Blur, Kalman Filter, Statistically Estimation, Badminton Shuttlecock.

\section{Introduction}

The research on visual object tracking for sports-events is conducted as application cases of computer vision and contributes to developing the tactics of games[1-7]. Because players and balls are the tracking target of the visual tracking processes, a visual tracking method that can handle multiple objects with fast and complicated movement is needed. Moreover, because a shuttlecock is a small (approximately $7 \mathrm{~cm}$ ) item, objects inside the video frame are observed in low resolution. For example, when we capture the game with a general-use video camera, the observation size in a frame might be just about a few pixels.

In this paper, we focus on a badminton shuttlecock as the tracking target since it has the cited problems conspicuously. A shuttlecock is composed of feathers of birds such as waterfowls, attached to the hemispheric cork with adhesive. Since it is much more lightweight than balls used for other games, attaching a transmitter or a marker for position sensing might be difficult. Such attachment would represent extra weight in the object causing trajectory changes. Thus, the tracking method using visual information is expected to be a promising solution to extract the trajectories. 


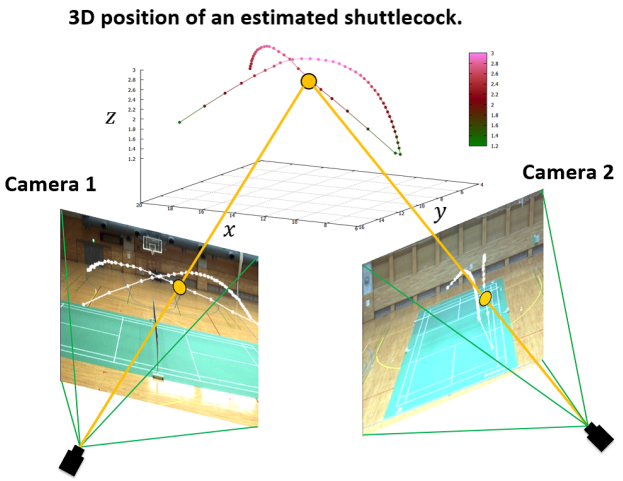

Fig. 1. The estimate result of the proposed method using a video sequence of a badminton game. (2 trajectories)

However, there is an additional problem to track the shuttlecock. Due to its structure, during the badminton game (rally) the moving velocity changes inconsistently and drastically during each rally due to the air resistance [8]. In order to solve the problem, we develop a tracking method that can get extractable information of the object motion depending on the motion speed. When an object moves with low velocity for the shutter-speed of a video camera, there is little motion blur in each frame and it is possible to estimate its accurate position. On the other hand, it is difficult to estimate the accurate position, when the shuttlecock moves fast because the motion blur occurs on one frame. In this case, however, we can estimate the velocity information by analyzing the shape of the motion blur region.

We utilize information provided by motion blur, and we propose a visual tracking method for an object that has variously and drastically changes its moving velocity. A summary of this method is shown in figure 2. Our method defines the shuttle's state by referring the velocity, for not only specifying the color class, but also switching the input information to Kalman filter. When the velocity is low, we observe only the position and estimate the velocity as the difference. At high velocity, we observe the only velocity, and estimate the position by integrating the velocity values. When the velocity is middle, we observe both of the position and velocity.

\section{Related Work}

Recently, several visual object tracking methods are actively developed using physical or probabilistic movement model. For example, Yang et. al.proposes a visual tracking method applying color and gradient orientation histogram features using a particle filter [17]. Another strategy applying Haar-like features and gradual adaboost through particle filter is proposed by Li et. al [18]. Vasileios et. al. improved the visual tracking precision by updating two observation points 


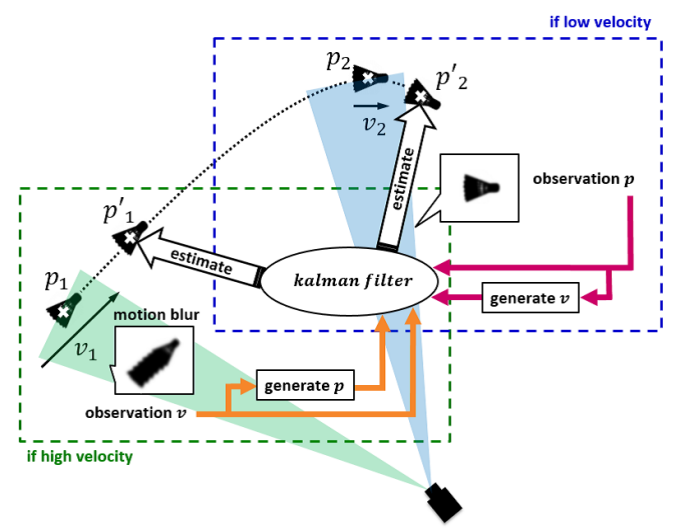

Fig. 2. A visual tracking method of an object that has variously and drastically changes its moving velocity by utilizing the motion blur. Because the observation precision of the position is high when the velocity is slow, we input to a Kalman filter "position (Observation position)" and "distance between the observed positions in the former and present frame (Observation velocity)". When the velocity is fast, we input to the Kalman filter "observed velocity (Observation velocity)" and "estimated position (Observation position)".

dynamically (1. An observation point calculated using only the Mean Shift in the observation point of the Kalman filter; 2. An observation point calculated from Mean Shift and the estimate point of the Kalman filter) [12]. Chang et al. separate into three the tracking level depending on the target sequence and proposes a visual tracking method inputting to the Kalman filter an observation value calculated per level [13]. Furthermore, recently, a visual tracking method that used a particle filter together with Kalman filter was studied. Satoh et. al. reduce the number of the particles in comparison with previous studies by using Kalman filter together in simple tracking method based on the information of the color, and succeed in the object tracking [14]. Xu et. al. use Kalman filter analytically and update the particles [15]. This way, the effectiveness of using a particle filter based on the probabilistic model together with the Kalman filter based on the physical motion model is appropriately shown. We follow this approach to improve the tracking accuracy. The motion of a shuttlecock can be expressed with a simple dynamics model and irregular motions due to turns or air resistance can be included using a probabilistic model.

If we insist on tracking the object just in the 2D image space, it becomes difficult when the target object is not observed in an image frame by occlusion. An effective solution is using images from multiple viewpoints $[11,16]$. In our approach, we also capture the target object by using at least two video cameras, and reconstruct the motion model in $3 \mathrm{D}$ space.

Usually, motion blur is regarded as a source of the observation error that reduces the accuracy of tracking process. On the other hand, there are studies that improve the accuracy by estimating motion blur from observing every frame 


\begin{tabular}{|c|c|c|c|c|c|c|c|c|}
\hline Camera & \multicolumn{4}{|c|}{ front } & \multicolumn{4}{|c|}{ side } \\
\hline Background court & & & & & & & & \\
\hline shuttle speed & high & low & high & low & high & low & high & low \\
\hline image & & & & $a$ & & a & & 6 \\
\hline
\end{tabular}

Fig. 3. Examples of the appearance of a badminton shuttlecock with motion blur (4pixels - 35pixels)

$[9,10,19]$. However, they do not directly use the motion blur as a source of object tracking, but restore the blurred image before the tracking process. Figure 3 shows how a fast moving object's position estimation accuracy will generally decrease because of the motion blur. However we understood that the velocity of the object could be directly estimated from the shape of the motion blur region.

\section{Badminton Shuttlecock Tracking Method Using Motion Blur}

In this paper, we tackle some issues of visual object tracking. First of all, the target object (shuttlecock) moves very fast. Second, the observed size of the shuttlecock is small. And third, the moving velocity changes inconsistently and drastically during each rally.

For the first issue, we propose a tracking method to estimate the moving velocity from the shape of the motion blur region. For the second issue, we renounce to calculate the likelihood from texture information such as gradient, and instead use the color information. Color information is affected by the environmental conditions such as lighting change and background color. So we generate a probability model of the observed shuttlecock's color to absorb the fluctuation. In the case of the third issue, we develop a tracking method that switches the estimating method depending on the velocity. When the velocity is slow, we input into Kalman filter the current observed position and as observed velocity, we input the distance between the positions observed in the former and present frames. When the velocity is fast, we input into Kalman filter the observed position and the observed velocity estimated by the shape of the motion blur.

\subsection{The Detection of a Moving Object Region}

At the beginning of the object tracking or after having lost sight of the shuttlecock, we detect the shuttlecock by the following processes. At first, moving object candidate regions are extracted by background subtraction processing. In this process player regions are excluded by referring to the player region size. In addition, we mask out the region such as a court-line and the net in where it is difficult to accurately perform the segmentation processing due to the high 


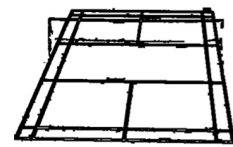

1

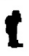

(a)

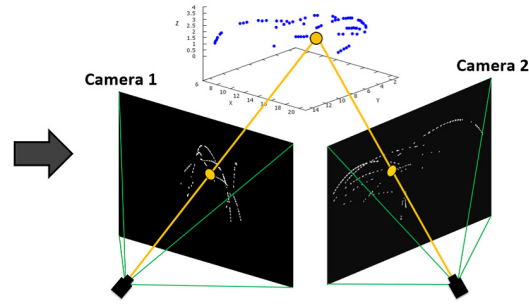

(b)

Fig. 4. (a) Masked out region for shuttlecock candidate. (b) Shuttlecock detection by using background subtraction method and the estimation of the 3D position.

brightness level. As shown in figure 4, we execute these processes in the frames captured from two viewpoints, and calculate the 3D position of the shuttlecock by stereo-vision. The 3D position of the shuttlecock is the observed position for Kalman filter.

\subsection{Construction of the Kalman Filter}

A 3D position, velocity, and acceleration are used for the state of the shuttlecock in the frame $k$.

$$
X_{k}=\left\{x_{k}, \dot{x}_{k}, \ddot{x}_{k}, y_{k}, \dot{y}_{k}, \ddot{y}_{k}, z_{k}, \dot{z}_{k}, \ddot{z}_{k}\right\}
$$

The state model of the Kalman filter is denoted by the Equation (2).

$$
X_{k}=A X_{k-1}+B u_{k}+\omega_{k}
$$

Here, $A$ is a state transition matrix, and the movement of the shuttlecock forms a parabola with the air resistance,

$$
A=\left[\begin{array}{ccccccccc}
1 & \delta_{t} & 0 & 0 & 0 & 0 & 0 & 0 & 0 \\
0 & 1 & \delta_{t} & 0 & 0 & 0 & 0 & 0 & 0 \\
0 & \frac{-c}{m} \delta_{t} & 0 & 0 & 0 & 0 & 0 & 0 & 0 \\
0 & 0 & 0 & 1 & \delta_{t} & 0 & 0 & 0 & 0 \\
0 & 0 & 0 & 0 & 1 & \delta_{t} & 0 & 0 & 0 \\
0 & 0 & 0 & 0 & \frac{-c}{m} \delta_{t} & 0 & 0 & 0 & 0 \\
0 & 0 & 0 & 0 & 0 & 0 & 1 & \delta_{t} & 0 \\
0 & 0 & 0 & 0 & 0 & 0 & 0 & 1 & \delta_{t} \\
0 & 0 & 0 & 0 & 0 & 0 & 0 & \frac{-c}{m} \delta_{t} & 0
\end{array}\right], B=\left[\begin{array}{cccc}
0 & \cdots & 0 & 0 \\
\vdots & \ddots & \vdots & \vdots \\
0 & \cdots & 0 & 0 \\
0 & \cdots & 0 & -g
\end{array}\right]
$$

$\delta_{t}$ is the time lag between two frames. $B u_{k}$ is a control input concerning a state transition. $m$ is mass and $c$ expresses the amount of air resistance. Since the acceleration due to gravity $g$ applied in the direction of $z$ is not included in the 
state transition matrix of $A$, The matrix $B$ is defined including this consideration. On the other hand, in the frame $k$, when the estimated $3 \mathrm{D}$ position of the shuttlecock is made into $z_{k}$, an observation model is expressed by the Equation (4), (5).

$$
\begin{aligned}
& z_{k}=\hat{H}_{k} X_{k}+\varepsilon_{k} \\
& \hat{H}_{k}=\left[\begin{array}{ccccccccc}
p_{x} & 0 & 0 & 0 & 0 & 0 & 0 & 0 & 0 \\
0 & v_{x} & 0 & 0 & 0 & 0 & 0 & 0 & 0 \\
0 & 0 & 0 & 0 & 0 & 0 & 0 & 0 & 0 \\
0 & 0 & 0 & p_{y} & 0 & 0 & 0 & 0 & 0 \\
0 & 0 & 0 & 0 & v_{y} & 0 & 0 & 0 & 0 \\
0 & 0 & 0 & 0 & 0 & 0 & 0 & 0 & 0 \\
0 & 0 & 0 & 0 & 0 & 0 & p_{z} & 0 & 0 \\
0 & 0 & 0 & 0 & 0 & 0 & 0 & v_{z} & 0 \\
0 & 0 & 0 & 0 & 0 & 0 & 0 & 0 & 0
\end{array}\right] \\
& \hat{H}_{k}=\left\{\begin{array}{l}
p_{\text {background-diff }}, v_{\text {interframe }}(\text { if LowSpeed }) \\
p_{\text {particle-center }}, v_{\text {blur }}(\text { if HighSpeed })
\end{array}\right. \\
& p \text { : position, } v \text { : velocity }
\end{aligned}
$$

An observation model defines a position and velocity. $\varepsilon_{k}$ is the random noise which occurs at the time of observation. The observation noise is a variance matrix computed from the observation error of the observation trajectory acquired manually and a trajectory without observation noise. According to the velocity gained by a process explained later ahead in this paper, the observation model $\hat{H}_{k}$ according to the object's velocity is obtained by choosing the observation information given to a Kalman filter.

\subsection{Likelihood Calculation Using the Information of the Color}

We calculate the likelihood of the tracked object using the information of the color. Figure 5(a) shows the distribution of the illuminance value in the observed badminton shuttlecock regions. The illuminance level of the shuttlecock is affected by the movement blur. Furthermore, the color of the shuttlecock looks mixed with the color of the background. Therefore, we divide the scenery of the badminton frame in two regions: the background and the court itself.

The influence of the movement blurring has a linear relation with velocity. Therefore, we classify the distribution into three classes ("fast" "middle velocity" "slow"), and we decide a likelihood model corresponding to each velocity. As clustering method, we employee k-means algorithm. As shown in Figure 5(a), the illuminance value of the shuttlecock observed in the court region and outside the court region are well segmented into two clusters. Figure 5(b) and (c) show the 
clustered results of the shuttlecock illuminance observed in the court region and outside the court region, respectively. In the figures, when the shuttlecock's speed is fast, it receives high influence from the motion blur, thus, the shuttlecock's color is seemingly mixed with the background color. In the figure's RGB space it is represented in the cyan-colored class. Similarly, when the shuttlecock is slow, as there is little influence of the motion blur, thus, the shuttlecock's color is observed as its original color. In the figure, it is represented in the pink-colored class. Finally, the middle velocity case is represented in the figure by the purplecolored class.

We assume the distance from the center of gravity of each colored-class and the actual shuttlecock's color as a likelihood function, and we decide to use six kinds of likelihood functions by the predictive position and velocity of the shuttlecock selectively. In addition, the predictive position of the shuttlecock judges whether the shuttlecock is observed in the court or the outside. The output formula of likelihood function $L(d)$ is presented in equation (6).

$$
L\left(d_{a, b}\right)=\frac{1}{\sqrt{2 \pi} \sigma} \exp \left(-\frac{\left(d_{a, b}\right)^{2}}{2 \sigma^{2}}\right)
$$

Likelihood function $L(d)$ is a function of Euclid distance $d$ from the center of gravity of each class. We assume that a normal distribution function becomes variance $\sigma^{2}$. The variance $\sigma^{2}$ sets it in reference to sample frame group. The likelihood function chooses $L\left(d_{a}\right)$, if the predictive position of the shuttlecock is the inside of the court. The outside likelihood function of the court considers is $L\left(d_{b}\right)$.

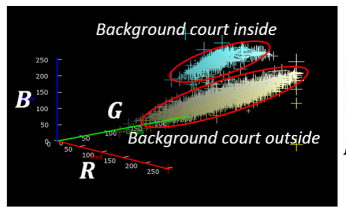

shuttle illuminance value

(a)

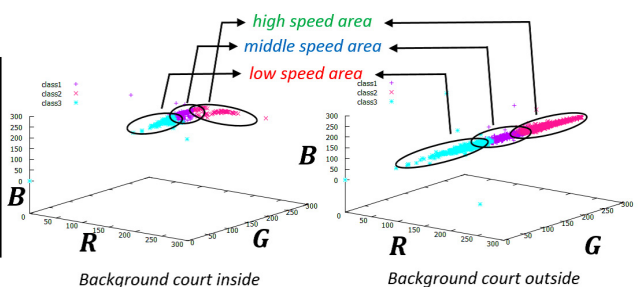

(c)

Fig. 5. (a)Distribution of the illuminance value of the observed badminton shuttlecock regions. (b) Clustering of the illuminance value of the shuttlecock observed in the court region. (c) Clustering of the illuminance value of the shuttlecock observed in the outside court region.

\subsection{Acquisition of the 3D Position and Velocity of the Object Using Particles}

Our method can statistically estimate the 3D position and velocity information of the object by using particles as shown in figure 6 . The particles are scattered 
around a 3D position predicted by Kalman filter. The initial variance is a range (spherical) of process noise $\omega_{k}$. The spherical variance range is transformed into an ellipsoid form figure 6(a) by using a velocity vector predicted by Kalman filter. It is possible to place a particle in the range where the motion blur has an effect by using a predictive velocity vector.

Then, the method repositions the particle as weighted by the output of the likelihood function figure 6(b). At this point, the particles in $3 \mathrm{D}$ space express the shape of the motion-blurred shuttlecock as shown in figure 6(c). The center of gravity of all particles is the 3D position of the shuttlecocks. We can acquire a velocity vector by analyzing the distribution of the relocation particle figure $6(\mathrm{~d})$ as equation (7). The movement velocity $v$ of the shuttlecock in the 3D position $g$ is calculated dividing the length of the major axis $l$ and the shutter-speed (opening time) $t$. Here, the length $l$ of the major axis of an ellipsoid formed by particles is the distance that the shuttlecock moves during the shutter opening time $t$ of the capturing camera.

$$
v=l / t
$$

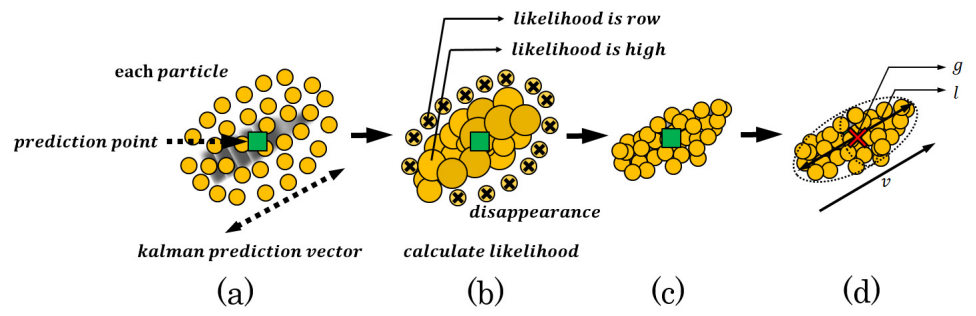

Fig. 6. (a) Transform a spherical distribution into an ellipsoid form. (b) Likelihood calculation, (c) Particle relocation, (d) Acquisition of a position and the velocity

\subsection{Likelihood Calculation of the Position of the Former Frame}

As the detection of the shuttlecock and construction of Kalman filter is impossible in the early frames after the shuttlecock has been hit, due to its fast movement, the visual tracking process mentioned above does not work. We solve this problem by rewinding the time-line, going from the initial observation frame (i.e., the first frame that the Kalman filter works) to former frame by using only the likelihood calculation (without Kalman filter prediction step). As shown in figure 7 , the position in time $(-t)$ can be tentatively predicted by the motion model of the shuttlecock described in section 3.1, and the position and velocity can be statistically estimated by using our method described in section 3.4.

In the frames just after the shuttlecock was hit, the motion blur is too strong to observe the position of the shuttlecock. As the result, the shuttlecock is not found by using particle scatters as shown in figure 8(a). In this case, we set 


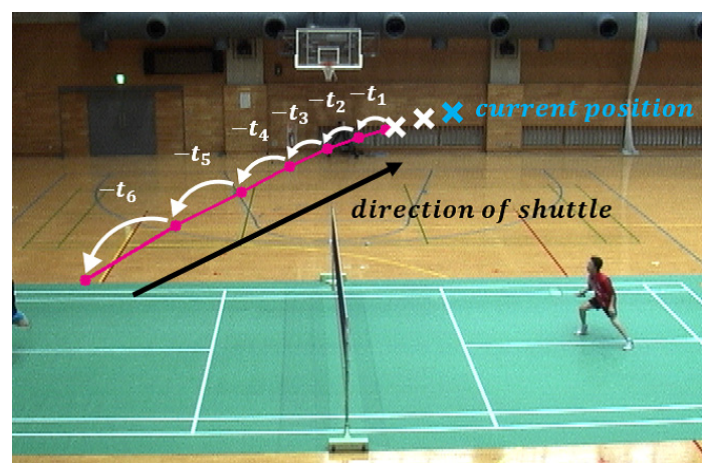

Fig. 7. Estimated Position in Time $-t_{k}$

a straight line between the last observed position (the shuttlecock was hit in the previous rally) and the most former position estimated by the time-rewind approach. Next the large particle scatter region along the extracted line is created (see Figure 8(b)). Because a lot of objects that have similar color exist in this region, the reliability of estimated position might be low. However the estimated position is useful for construction of the Kalman filter.

By using this approach it is possible to estimate the position of the object with variations in sizes of the blur region.

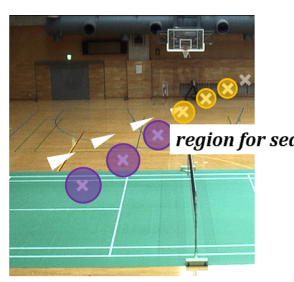

(a)

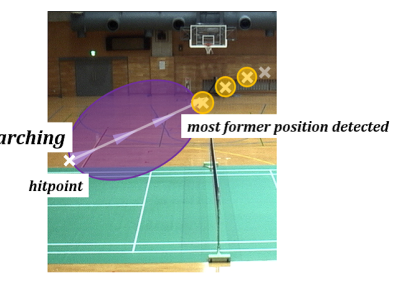

(b)

Fig. 8. (a) Illustration then the shuttlecock is not found by the particle scatters. (b) The large particle scatters region along the line between hit point and firstly detected position of the shuttlecock.

\section{Experiment}

We evaluate the effectiveness of the proposed method by using the video sequences synchronously captured using two video cameras. We capture the videos by using SONY BRC-300 cameras with $640 \times 480$ resolution, 30fps, and 1/60 sec shutter speed. The test video sequence includes a rally of the badminton game. 


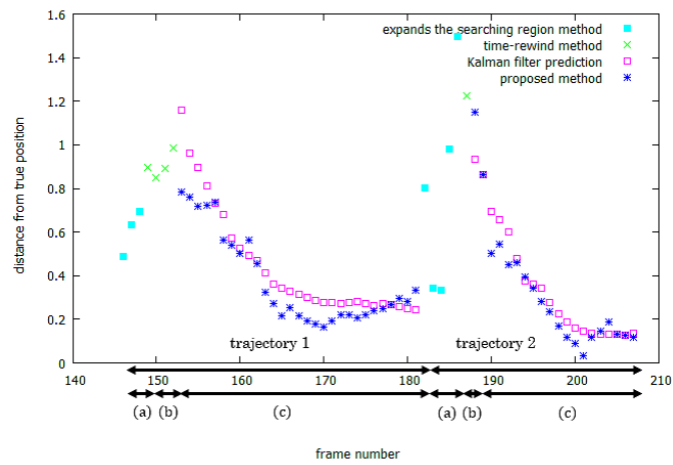

Fig. 9. The error of the position estimation across the frame

Table 1. The error reduction rate by our proposed method

\begin{tabular}{l|l|l|l}
\hline & $\begin{array}{l}\square \text { prediction error } \\
\text { average }[\mathrm{m}]\end{array}$ & $\begin{array}{l}\text { *proposal method } \\
\text { error average }[\mathrm{m}]\end{array}$ & reduction rate \\
\hline Trajectory1 & 0.44 & 0.37 & $18 \%$ \\
\hline Trajectory2 & 0.35 & 0.32 & $10 \%$ \\
\hline Trajectory1,2average & 0.40 & 0.34 & $14 \%$ \\
\hline
\end{tabular}

Figure 9 shows the estimation error calculated during 2 strokes. The estimation error of the predicted position by Kalman filter is plotted as " $\square$ ", and the estimated error by the proposed method is "*". The Error reduction rate by our method is shown in the table 1 . We can see that our method, with the motion blur, improves the estimation results compare with the Kalman filter. However, when the number of the observations is not sufficient the Kalman filter cannot construct. For example the visual tracking by using Kalman filter does not work at the frame 146-152, and 182-187.

The " $\times$ " expresses the error of the estimated position by the time-rewind approach. As the result, we can confirm that our method described in section 3.5 well estimates the shuttlecock position at the frame 149-152, 187. The range of the error is approximately less than $1 \mathrm{~m}$. However, a visual tracking is still difficult when the shuttlecock is not observed in the large particle scatters region as shown at frames 146-148,182-186. Therefore, we expand the search region as described in section 3.5, it at frames 146-148, 182-186. The "ם" expresses the error of the estimated position by the method that expands the searching region. By using rewinding the time-line method (denoted by " $\times$ ") we lower the error. The error range is approximately less than $1.5 \mathrm{~m}$.

\section{Conclusion}

In this paper, we proposed a visual object tracking and trajectory estimation method that is robust to low observing resolution, and wide range of the variation 
of the target object velocity. The key-idea behind our method is the use of the motion blur region characteristic for observing the moving velocity of the target object. Furthermore, our method is capable to estimate the position of the shuttlecock during very fast motion by rewinding the time sequence.

We conducted on experiments to confirm the effectiveness of the proposed visual tracking and trajectory estimation method. We confirmed that the observation using the particle could improve the estimation error by about $14 \%$ compared with the ordinary tracking method by Kalman filter (see Table 1).

\section{References}

1. Gandhi, H., Collins, M., Chuang, M., Narasimhan, P.: Real-Time Tracking of Game Assets in American Football for Automated Camera Selection and Motion Capture. Procedia Engineering 2(2), 2667-2673 (2010)

2. Lu, W.-L., Okuma, K., Little, J.J.: Tracking and recognizing actions of multiple hockey players using the boosted particle filter. Image and Vision Computing 27(12), 189-205 (2009)

3. Chen, H.-T., Tien, M.-C., Chen, Y.-W., Tsai, W.-J., Lee, S.-Y.: Physics-based ball tracking and $3 \mathrm{D}$ trajectory reconstruction with applications to shooting location estimation in basketball video. J. Vis. Commun. Image R 20(3), 204-216 (2009)

4. Chen, H.-T., Chen, H.-S., Hsiao, M.-H., Chen, Y.-W., Lee, S.-Y.: A TrajectoryBased Ball Tracking Framework with Enrichment for Broadcast Baseball Videos. In: International Computer Symposium (ICS 2006), Taiwan, vol. III, pp. 1145-1150 (December 2006)

5. Yan, F., Christmas, W., Kittler, J.: Layered Data Association Using GraphTheoretic Formulation with Application to Tennis Ball Tracking in Monocular Sequences. IEEE Transactions on Pattern Analysis and Machine Intelligence 30(10), 1814-1830 (2008)

6. Ren, J., Orwell, J., Jones, G.A., Xu, M.: Tracking the soccer ball using multiple fixed cameras. Computer Vision and Image Understanding 113(5), 633-642 (2009)

7. Chen, H.-T., Tsai, W.-J., Lee, S.-Y.: Ball tracking and 3D trajectory approximation with applications to tactics analysis from single-camera volleyball sequences. Multimedia Tools and Applications 60(3), 641-667 (2012)

8. Alam, F., Chowdhury, H., Theppadungporn, C., Subic, A.: Measurements of Aerodynamic Properties of Badminton Shuttlecocks. Procedia Engineering 2(2), 2487$2492(2010)$

9. Jin, H., Favaro, P., Cipolla, R.: Visual Tracking in the Presence of Motion Blur. In: Computer Vision and Pattern Recognition (CVPR 2005), vol. 2, pp. 18-25 (June 2005)

10. Park, Y., Lepetit, V., Woo, W.: Handling Motion-Blur in 3D Tracking and Rendering for Augmented Reality. IEEE Transactions on Visualization and Computer Graphics (TVCG) 18(9), 1449-1459 (2012)

11. Wu, Z., Hristov, N.I., Hedrick, T.L., Kunz, T.H., Betke, M.: Tracking a Large Number of Objects from Multiple Views. In: IEEE 12th International Conference on Computer Vision (ICCV 2009), pp. 1546-1553 (September-October 2009)

12. Karavasilis, V., Nikou, C., Likas, A.: Visual Tracking by Adaptive Kalman Filtering and Mean Shift. In: Konstantopoulos, S., Perantonis, S., Karkaletsis, V., Spyropoulos, C.D., Vouros, G. (eds.) SETN 2010. LNCS, vol. 6040, pp. 153-162. Springer, Heidelberg (2010) 
13. Huang, C., Wu, B., Nevatia, R.: Robust Object Tracking by Hierarchical Association of Detection Responses. In: Forsyth, D., Torr, P., Zisserman, A. (eds.) ECCV 2008, Part II. LNCS, vol. 5303, pp. 788-801. Springer, Heidelberg (2008)

14. Satoh, Y., Okatani, T., Deguchi, K.: A Color-based Tracking by Kalman Particle Filter. In: International Conference on Pattern Recognition (ICPR 2004), vol. 3, pp. 502-505 (August 2004)

15. Xu, X., Li, B.: Adaptive Rao-Blackwellized Particle Filter and Its Evaluation for Tracking in Surveillance. IEEE Transactions on Image Processing 16(3), 838-849 (2007)

16. Reilly, V., Idrees, H., Shah, M.: Detection and Tracking of Large Number of Targets in Wide Area Surveillance. In: Daniilidis, K., Maragos, P., Paragios, N. (eds.) ECCV 2010, Part III. LNCS, vol. 6313, pp. 186-199. Springer, Heidelberg (2010)

17. Yang, C., Duraiswami, R., Davis, L.: Fast Multiple Object Tracking via a Hierarchical Particle Filter. In: IEEE International Conference on Computer Vision (ICCV 2005), vol. 1, pp. 212-219 (October 2005)

18. Li, Y., Ai, H., Yamashita, T., Lao, S., Kawade, M.: Tracking in Low Frame Rate Video: A Cascade Particle Filter with Discriminative Observers of Different Lifespans. IEEE Transactions on Pattern Analysis and Machine Intelligence 30(10), 1728-1740 (2008)

19. Wu, Y., Ling, H., Yu, J., Li, F., Mei, X., Cheng, E.: Blurred Target Tracking by Blur-driven Tracker. In: IEEE International Conference on Computer Vision (ICCV), pp. 1100-1107 (2011) 